\title{
All-optical production of a lithium quantum gas using narrow-line laser cooling
}

\author{
P. M. Duarte, R. A. Hart, J. M. Hitchcock, ${ }^{*}$ T. A. Corcovilos, ${ }^{\dagger}$ T.-L. Yang, A. Reed, ${ }^{\ddagger}$ and R. G. Hulet \\ Department of Physics and Astronomy and Rice Quantum Institute, Rice University, Houston, Texas 77005, USA
}

(Received 23 September 2011; published 21 December 2011)

\begin{abstract}
We have used the narrow $2 S_{1 / 2} \rightarrow 3 P_{3 / 2}$ transition in the ultraviolet (uv) to laser cool and magneto-optically trap (MOT) ${ }^{6} \mathrm{Li}$ atoms. Laser cooling of lithium is usually performed on the $2 S_{1 / 2} \rightarrow 2 P_{3 / 2}$ (D2) transition, and temperatures of $\sim 300 \mu \mathrm{K}$ are typically achieved. The linewidth of the uv transition is seven times narrower than the D2 line, resulting in lower laser cooling temperatures. We demonstrate that a MOT operating on the uv transition reaches temperatures as low as $59 \mu \mathrm{K}$. Furthermore, we find that the light shift of the uv transition in an optical dipole trap at $1070 \mathrm{~nm}$ is small and blueshifted, facilitating efficient loading from the uv MOT. Evaporative cooling of a two spin-state mixture of ${ }^{6} \mathrm{Li}$ in the optical trap produces a quantum degenerate Fermi gas with $3 \times 10^{6}$ atoms in a total cycle time of only $11 \mathrm{~s}$.
\end{abstract}

DOI: 10.1103/PhysRevA.84.061406

PACS number(s): 37.10.De, 32.10.Dk, 67.85.Lm

The creation of quantum degenerate gases using all-optical techniques [1-4] offers several advantages over methods employing magnetic traps. Optical potentials can trap any ground state, allowing selection of hyperfine sublevels with favorable elastic and inelastic scattering properties. In the case of Fermi gases, the ability to trap atoms in more than one sublevel eliminates the need for sympathetic cooling with another species [5,6], greatly simplifying the experimental setup. All-optical methods also facilitate rapid evaporative cooling since magnetically tunable Feshbach resonances can be employed to achieve fast thermalization.

There are, however, challenges to all-optical methods. An essential prerequisite is an optical potential whose depth is sufficiently greater than the temperature of the atoms being loaded. The usual starting point is a laser cooled atomic gas confined to a magneto-optical trap (MOT). In a twolevel picture, atoms may be cooled to the Doppler limit $T_{D}=\hbar \Gamma /\left(2 k_{B}\right)$, where $\Gamma /(2 \pi)$ is the natural linewidth of the excited state of the cooling transition $[7,8]$. In many cases, however, sub-Doppler temperatures can be realized due to the occurrence of polarization gradient cooling arising from the multilevel character of real atoms [9]. Polarization gradient cooling mechanisms are effective if the linewidth of the cooling transition is small compared to the hyperfine splitting of the excited state, or if there is a large degree of magnetic degeneracy in the ground state [10]. The limit to cooling in these cases is the recoil temperature $T_{R}=\hbar^{2} k^{2} /\left(2 m k_{B}\right)$, where $k$ is the wave number of the laser cooling transition and $m$ is the mass of the atom.

Polarization gradient cooling is found to be efficient for most of the alkali-metal atoms including $\mathrm{Na}, \mathrm{Rb}$, and Cs; MOTs of these species routinely attain temperatures of $\sim 10 \mu \mathrm{K}$, which is not far above $T_{R}$. Unfortunately, for $\mathrm{Li}$ and $\mathrm{K}$, the elements most often employed in Fermi-gas

\footnotetext{
*Present address: Thermo Fisher Scientific, 2215 Grand Avenue Pkwy., Austin, TX 78728.

${ }^{\dagger}$ Present address: Department of Physics, The Pennsylvania State University, 104 Davey Laboratory, University Park, PA 16802.

${ }^{\ddagger}$ Present address: Department of Physics, University of Colorado, 2000 Colorado Ave., Boulder, CO 80309.
}

experiments, sub-Doppler cooling is ineffective in the presence of magnetic fields, including those required for a MOT. For $\mathrm{Li}$, sub-Doppler cooling is inhibited because the hyperfine splitting of the excited state is unresolved (Fig. 1), thus limiting temperatures to $\sim 300 \mu \mathrm{K}$, roughly twice the Doppler limit. Sub-Doppler cooling has been attained in both the bosonic $[11,12]$ and fermionic [13] isotopes of $\mathrm{K}$ by going from a MOT to a molasses phase, and appropriately ramping the laser parameters $[11,12]$. While this can work at high densities for the bosonic isotopes [11], in the case of fermionic ${ }^{40} \mathrm{~K}$ the lower temperatures are achieved at the expense of density [13], which is not advantageous for loading and evaporative cooling in a trap.

Laser cooling has also been demonstrated with atoms possessing ultranarrow, dipole-forbidden transitions, such as the intercombination lines of the alkaline-earth-metal atoms, where $T_{D} \lesssim T_{R}$. These experiments produce temperatures in the range of $1-10 \mu \mathrm{K}$ [15-18]. In this Rapid Communication, we report a similar strategy for ${ }^{6} \mathrm{Li}$, where we form a MOT using the narrow, yet still dipole-allowed, $2 S_{1 / 2} \rightarrow$ $3 P_{3 / 2}$ transition shown in Fig. 1. The narrower linewidth of this transition compared to the usual D2 line gives a correspondingly lower Doppler temperature. Perhaps just as significant for reaching high phase space densities as a prelude to evaporative cooling is that the shorter wavelength of this transition reduces the optical depth and radiation trapping, which are important density limiting effects [19]. We demonstrate the effectiveness of this laser cooling scheme as the starting point for efficiently loading an optical dipole trap and producing a degenerate Fermi gas with a large atom number. The advantage of using a uv transition to obtain high density was previously demonstrated with metastable helium [20]. In that case, however, the transition linewidth was identical with the conventionally used transition, and the temperature was not reduced. Also, the analogous narrow, but dipole-allowed, $4 S_{1 / 2} \rightarrow 5 P_{3 / 2}$ transition in $\mathrm{K}$ has been used recently to achieve lower temperatures in a MOT [21].

The experimental sequence begins by loading a conventional red MOT, operating on the $2 S_{1 / 2} \rightarrow 2 P_{3 / 2}$ (D2) transition at $671 \mathrm{~nm}[5,6,22]$, from a laser slowed atomic beam. Each of the six MOT beams consists of light resonant with transitions between the $F=3 / 2$ and $F=1 / 2$ hyperfine 


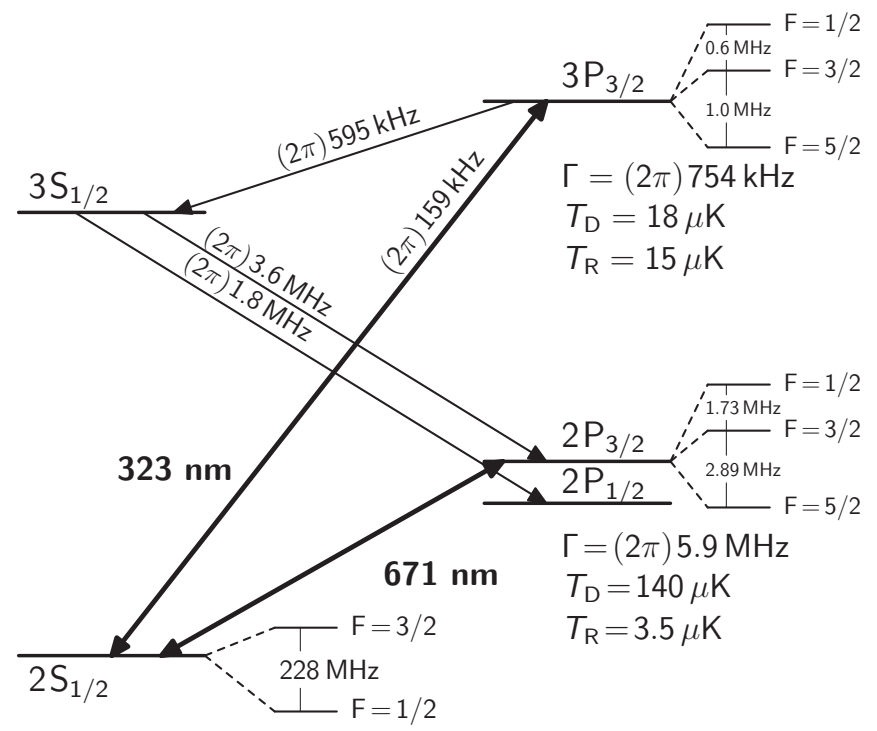

FIG. 1. Partial energy-level diagram of ${ }^{6} \mathrm{Li}$ showing relevant transitions. Lines in bold represent the transitions used to laser cool atoms. Lighter lines represent decay pathways from the excited $3 P_{3 / 2}$ state. The corresponding decay rates are indicated [14].

ground states and the excited $2 P_{3 / 2}$ state, which we refer to as the cooling and repumping beams, respectively. These beams have $1 / e^{2}$ radii of $0.9 \mathrm{~cm}$ and peak intensities per beam of $1.3 I_{\mathrm{sat}}^{2 P}$ on the cooling transition and $0.4 I_{\mathrm{sat}}^{2 P}$ on the repumping transition, where $I_{\text {sat }}^{2 P}=5.1 \mathrm{~mW} / \mathrm{cm}^{2}$ is the saturation intensity for the red transition. After $\sim 5 \mathrm{~s}$ of loading, we collect $N \simeq 1.5 \times 10^{9}$ atoms at $T \simeq 1 \mathrm{mK}$ and a peak density $n_{0} \simeq 2.4 \times 10^{10} \mathrm{~cm}^{-3}$. We then cool and compress the red MOT by simultaneously reducing the intensity and detuning of the cooling and repumping light and increasing the magnetic field gradient. At the end of this stage, indicated as CMOT in Fig. 2(a), the relevant quantities are $N \simeq 1 \times$ $10^{9}$ atoms, $T \simeq 290 \mu \mathrm{K}$, and $n_{0} \simeq 3.4 \times 10^{10} \mathrm{~cm}^{-3}$.

The six beams for the uv MOT are overlapped with the red MOT beams using dichroic mirrors. The required uv light is generated by a frequency-doubled laser system consisting of a grating stabilized diode laser operating at $646 \mathrm{~nm}$, a tapered amplifier, and an external ring doubling cavity [23]. The output power at $323 \mathrm{~nm}$ is limited to $30 \mathrm{~mW}$, but the total power incident at the atoms is only $11 \mathrm{~mW}$ due to power splitoff for frequency stabilization and to losses from optics and vacuum viewports. The cooling (repumping) beams of the uv MOT have $1 / e^{2}$ radii of $0.33 \mathrm{~cm}(0.40 \mathrm{~cm})$ and peak intensities per beam of $0.2 I_{\text {sat }}^{3 P}\left(0.02 I_{\text {sat }}^{3 P}\right)$, where $I_{\text {sat }}^{3 P}=27.7 \mathrm{~mW} / \mathrm{cm}^{2}$ is the saturation intensity for the uv transition. Atoms are loaded into the uv MOT by abruptly reducing the magnetic field gradient, turning off the red MOT light, and turning on the uv MOT light, as shown in Fig. 2(a). A small magnetic field gradient during the $1.25 \mathrm{~ms}$ loading phase increases the effective capture volume, and a large uv laser detuning helps capture high-velocity atoms from the red MOT. The uv MOT captures $\sim 5 \times 10^{8}$ atoms, corresponding to an efficiency of $50 \%$. The loss of atoms is most likely due to small uv beam waists, which are limited by the total available power at $323 \mathrm{~nm}$. Following the loading phase the magnetic field gradient is increased linearly, and the uv detuning is reduced
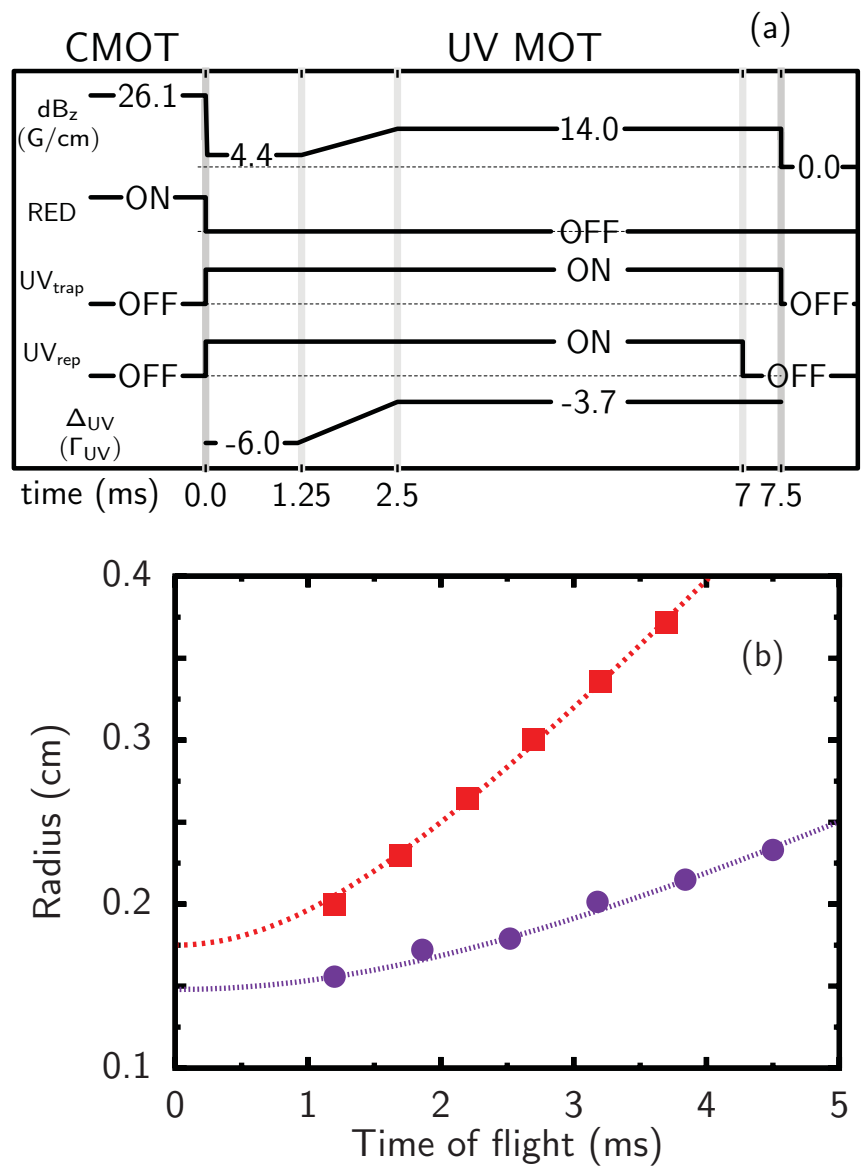

FIG. 2. (Color online) (a) Timing diagram of the transfer sequence from the CMOT to the uv MOT. (b) Time-of-flight expansion of atoms released from red and uv MOTs. The red squares (violet circles) represent the $1 / e$ radius of Gaussian fits to the spatial profile of freely expanding clouds of atoms released from the CMOT (uv MOT). The lines are fits to ballistic expansions which give temperatures of 288 and $59 \mu \mathrm{K}$ and corresponding phase space densities of $2.5 \times 10^{-6}$ and $2.3 \times 10^{-5}$ for the CMOT and uv MOT, respectively.

over a period of $1.25 \mathrm{~ms}$. The values of this steady-state uv MOT are chosen to optimize loading into the optical dipole trap, as described below. After a hold time of $5 \mathrm{~ms}$, the temperature is measured in time of flight by releasing and subsequently imaging the atoms by pulsing on the red MOT cooling and repumping light at full intensity for $0.1 \mathrm{~ms}$ while recording the fluorescence on a CCD camera.

Figure 2(b) shows a comparison of the performance of the red CMOT and the uv MOT. For the uv MOT, $T=59 \mu \mathrm{K}$ and $n_{0} \simeq 2.9 \times 10^{10} \mathrm{~cm}^{-3}$. This corresponds to a phase space density $\rho_{\mathrm{ps}}=n_{0}\left(h / \sqrt{2 \pi m k_{B} T}\right)^{3}=2.3 \times 10^{-5}$, which is an order of magnitude higher than that produced by the CMOT and thus provides a significantly improved starting point for loading into an optical dipole trap and subsequent evaporative cooling. Lower temperatures may be attained in the uv MOT but only at the cost of reducing the peak density. Similarly, the density may be increased by using higher magnetic field gradients and larger detunings of the uv cooling light, but this results in higher $T$ and lower phase space density. We also investigated a uv MOT in which the repumping was done with the red transition. In this case, the temperature was the same 
as that obtained with uv repumping, but only half as many atoms remained in the uv MOT and at a smaller density of $n_{0} \simeq 2.0 \times 10^{10} \mathrm{~cm}^{-3}$.

The light for the optical dipole trap is provided by a broadband fiber laser operating at $1070 \mathrm{~nm}$ with a nominal output power of $50 \mathrm{~W}$. The trap consists of a single $38 \mathrm{~W}$ beam passed through the vacuum chamber and then reintroduced to the chamber at an angle of $15^{\circ}$ with respect to the first pass and with orthogonal polarization. Each beam is cylindrically symmetric and focused to a $1 / e^{2}$ radius of $73 \mu \mathrm{m}$ at the point of intersection. The trap depth per beam $U$ is $280 \mu \mathrm{K}$, and the radial and axial frequencies of the trap are measured to be $\omega_{r}=(2 \pi) 3.8 \mathrm{kHz}$ and $\omega_{z}=(2 \pi) 475 \mathrm{~Hz}$, respectively.

Atoms are loaded into the optical dipole trap by quickly turning on the trap light when the steady-state values of the uv MOT have been reached, at $2.5 \mathrm{~ms}$ in the timing diagram of Fig. 2(a). We find that laser cooling on the uv transition is effective in the trap, and that loading is improved by leaving the uv MOT on for $5 \mathrm{~ms}$ following turn on of the trap. The repumping light is turned off during the last $0.5 \mathrm{~ms}$ of loading, causing the atoms to be optically pumped into the $F=1 / 2$, $m_{F}= \pm 1 / 2$ hyperfine ground states, which we label as states $|1\rangle$ and $|2\rangle$. The uv light and magnetic field gradient are then abruptly switched off. A bias magnetic field is ramped within the next $20 \mathrm{~ms}$ to $330 \mathrm{G}$, where the scattering length is $\sim-280 a_{o}$. We perform evaporative cooling at $330 \mathrm{G}$ instead of near the Feshbach resonance at $834 \mathrm{G}$ because we observe density-dependent loss in the unitary scattering regime that is fast enough to reduce the efficiency of evaporation [26]. This loss is unobservable at $330 \mathrm{G}$.

The evaporation trajectory is shown in Fig. 3. We leave the dipole trap at full power for $200 \mathrm{~ms}$ following loading to allow

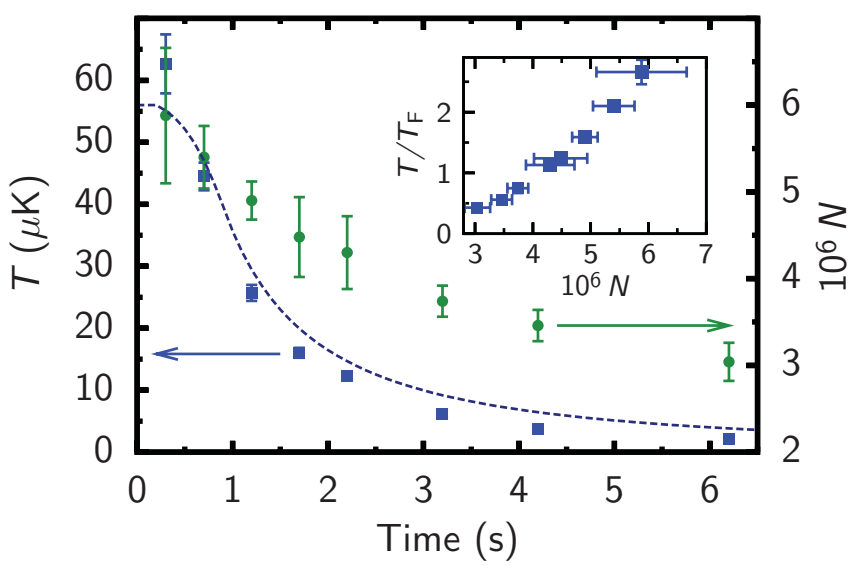

FIG. 3. (Color online) Number $N$ (green circles) and temperature $T$ (blue squares) of atoms in the optical trap during forced evaporation. Error bars for $N$ are one standard deviation of the mean value of $N$ for a sample of five measurements. The dotted blue line is $U / 5$, indicating the evaporation trajectory. The inset shows $T / T_{F}$ for the points in the main plot. For $T / T_{F}<1$ a surface fit to a polylog $[24,25]$ was used to determine $T / T_{F}$; otherwise, $T$ was measured by ballistic expansion and $T_{F}$ obtained from the mean value of $N$ and the measured trap frequencies. Systematic uncertainties in all measured quantities are estimated to be $10 \%$. for free evaporation of the atoms. At this point $6 \times 10^{6}$ atoms remain at $T=60 \mu \mathrm{K}$, corresponding to $T / T_{F} \approx 2.7$, where $T_{F}=\hbar \bar{\omega}(3 N)^{1 / 3}$ and $\bar{\omega}=\left(\omega_{r}^{2} \omega_{z}\right)^{1 / 3}$. With these parameters the peak density is $n_{0}=2.7 \times 10^{13} \mathrm{~cm}^{-3}$. These are excellent starting conditions for forced evaporation, which is performed by reducing the trap laser power. After $6 \mathrm{~s}$ of forced evaporation to $U=19 \mu \mathrm{K}$, a degenerate sample is obtained with $3 \times 10^{6}$ atoms at $T / T_{F}=0.45$.

A key to the performance of this scheme is the ability of the uv MOT to achieve both low laser-cooled temperatures and high densities. The number of atoms loaded into the dipole trap is predicted to depend exponentially on the ratio of the depth of the dipole potential to the MOT temperature [27]. Although the optical dipole trap utilizes a high-power laser, its depth is only $280 \mu \mathrm{K}$. Such a depth would be ill suited to capture atoms from the red MOT. In that case, either considerably greater light power or a smaller trap volume would be needed.

Also intrinsic to the capability of the uv MOT to load atoms into the dipole trap is the ability to continue to laser cool on the uv transition in the optical trap, which is possible only if the differential ac Stark shift of the $2 S_{1 / 2}$ and $3 P_{3 / 2}$ states produced by the dipole trap light is sufficiently small [28,29]. Otherwise, the light shift would prevent uniform laser cooling in the trap, and depending on its sign, could even cause heating. Our dipole trap operates at $1070 \mathrm{~nm}$ near a predicted "magic wavelength" for the uv transition [30], where the differential Stark shift vanishes.

We measured the differential ac Stark shift of the uv cooling transition by performing spectroscopy in the optical dipole trap. The atoms are first cooled to $T=3.5 \mu \mathrm{K}$ by $6 \mathrm{~s}$ of forced evaporation. Following evaporation, the optical trap is adiabatically ramped to various peak intensities, and the magnetic field is then quickly ramped to zero where the atoms are illuminated by uv light with a frequency tuned near the $2 S_{1 / 2}, F=1 / 2 \rightarrow 3 P_{3 / 2}$ transition. Resonant excitation

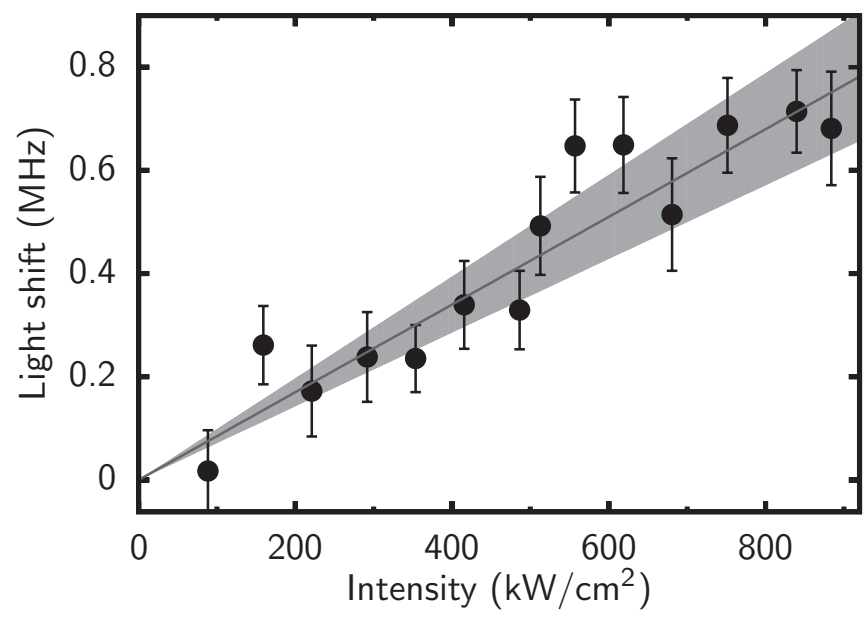

FIG. 4. Differential ac Stark shift of the $2 S_{1 / 2} \rightarrow 3 P_{3 / 2}$ transition as a function of intensity of the optical trapping light at $\lambda=1070 \mathrm{~nm}$. The circles represent the center of a Gaussian fit of a loss resonance (see text) and the error bars are $1 \sigma$ statistical error of these fits. The solid line is a linear fit to the resonance position with a slope of $850(140) \mathrm{Hz} /\left(\mathrm{kW} / \mathrm{cm}^{2}\right)$, where the uncertainty (shown by gray shading) represents the statistical uncertainty of the fit and a systematic uncertainty of $10 \%$ on the value of the trap intensity. 
causes atoms to be optically pumped out of the $F=1 / 2$ ground state. The population remaining in $|2\rangle$ is subsequently measured by absorption imaging at a field of $530 \mathrm{G}$. Spectra are recorded at several trap intensities, and the center of each is found by fitting to a Gaussian. These resonance locations are displayed as a function of peak intensity in Fig. 4. We find that at full trap depth, the uv transition is shifted to a $750 \mathrm{kHz}$ greater frequency than for free space. This is consistent with our observation that atoms are most efficiently loaded from the uv MOT when the uv laser frequency is shifted to the blue by approximately one linewidth. With this detuning the temperature of atoms loaded into the optical trap is $70 \mu \mathrm{K}$, close to the temperature in the MOT. The fact that the light shift is small and to the blue ensures that laser cooling is effective throughout the trap volume.

We have created a degenerate two-component Fermi gas with $3 \times 10^{6}{ }^{6} \mathrm{Li}$ atoms in $11 \mathrm{~s}$ using all-optical methods. Our results demonstrate that laser cooling on a narrow, but still dipole-allowed, uv transition substantially increases the atom number and production rate of a quantum degenerate gas. Three features contribute to the success of this method: (1) The narrow linewidth gives lower temperatures, enabling trapping with lower optical trap depth, and hence, a larger trap volume for a given laser power; (2) the differential light shift at the trapping wavelength is both small and to the blue, which greatly enhances loading by permitting laser cooling to proceed in the presence of the optical trap [29]; and (3) the short wavelength cooling transition allows laser cooling to be effective even at higher densities. Since $T_{R} \simeq T_{D}$ for the uv transition, the linewidth is sufficiently broad to avoid the need for either a spectrally broadened source or a "quench" laser to effectively broaden an ultranarrow transition by coupling it to a faster decaying excited state [16,17,29]. Additionally, since $T_{D}$ for the $\mathrm{D} 2$ line is only seven times larger than $T_{D}$ for the uv transition, transfer from the red to uv MOT proceeds efficiently without the need of an intermediate cooling stage $[15,18]$. The generation of Fermi gases with large number and densities is expected to be important in optical lattice experiments that require unit filling, including the search for antiferromagnetic order in the Hubbard model [31].

We thank Tom Killian and Joseph Thywissen for useful discussions. We also acknowledge contributions to the experiment by Kevin Jourde, Adrien Signoles, and Florian Emaury. This work was supported under ARO Grant No. W911NF-07-1-0464 with funds from the DARPA OLE program, the AFOSR DURIP program, NSF, ONR, and the Welch Foundation (Grant No. C-1133).
[1] M. D. Barrett, J. A. Sauer, and M. S. Chapman, Phys. Rev. Lett. 87, 010404 (2001).

[2] S. R. Granade, M. E. Gehm, K. M. O'Hara, and J. E. Thomas, Phys. Rev. Lett. 88, 120405 (2002); K. M. O’Hara, S. L. Hemmer, M. E. Gehm, S. R. Granade, and J. E. Thomas, Science 298, 2179 (2002).

[3] T. Weber, J. Herbig, M. Mark, H.-C. Nägerl, and R. Grimm, Science 299, 232 (2003).

[4] S. Jochim, M. Bartenstein, A. Altmeyer, G. Hendl, S. Riedl, C. Chin, J. Hecker Denschlag, and R. Grimm, Science 302, 2101 (2003).

[5] A. G. Truscott, K. E. Strecker, W. I. McAlexander, G. B. Partridge, and R. G. Hulet, Science 291, 2570 (2001).

[6] F. Schreck, L. Khaykovich, K. L. Corwin, G. Ferrari, T. Bourdel, J. Cubizolles, and C. Salomon, Phys. Rev. Lett. 87, 080403 (2001).

[7] T. Hänsch and A. Schawlow, Opt. Commun. 13, 68 (1975).

[8] D. Wineland and H. Dehmelt, Bull. Am. Phys. Soc. 20, 637 (1975).

[9] S. Chu, Rev. Mod. Phys. 70, 685 (1998); C. N. Cohen-Tannoudji, ibid. 70, 707 (1998); W. D. Phillips, ibid. 70, 721 (1998).

[10] X. Xu, T. H. Loftus, J. W. Dunn, C. H. Greene, J. L. Hall, A. Gallagher, and J. Ye, Phys. Rev. Lett. 90, 193002 (2003).

[11] M. Landini, S. Roy, L. Carcagní, D. Trypogeorgos, M. Fattori, M. Inguscio, and G. Modugno, Phys. Rev. A 84, 043432 (2011).

[12] V. Gokhroo, G. Rajalakshmi, R. K. Easwaran, and C. S. Unnikrishnan, J. Phys. B 44, 115307 (2011).

[13] G. Modugno, C. Benkő, P. Hannaford, G. Roati, and M. Inguscio, Phys. Rev. A 60, R3373 (1999).

[14] Y. Ralchenko, A. Kramida, J. Reader, and N. A. Team, NIST Atomic Spectra Database (ver. 4.1.0), 2011 November 23, National Institute of Standards and Technology, Gaithersburg, $\mathrm{MD}$, available at [http://physics.nist.gov/asd].
[15] H. Katori, T. Ido, Y. Isoya, and M. Kuwata-Gonokami, Phys. Rev. Lett. 82, 1116 (1999).

[16] T. Binnewies, G. Wilpers, U. Sterr, F. Riehle, J. Helmcke, T. E. Mehlstäubler, E. M. Rasel, and W. Ertmer, Phys. Rev. Lett. 87, 123002 (2001).

[17] E. A. Curtis, C. W. Oates, and L. Hollberg, Phys. Rev. A 64, 031403 (2001).

[18] T. H. Loftus, T. Ido, A. D. Ludlow, M. M. Boyd, and J. Ye, Phys. Rev. Lett. 93, 073003 (2004).

[19] D. W. Sesko, T. G. Walker, and C. E. Wieman, J. Opt. Soc. Am. B 8, 946 (1991).

[20] A. S. Tychkov, J. C. J. Koelemeij, T. Jeltes, W. Hogervorst, and W. Vassen, Phys. Rev. A 69, 055401 (2004).

[21] D. C. McKay, D. Jervis, D. J. Fine, J. W. Simpson-Porco, G. J. A. Edge, and J. H. Thywissen, Phys. Rev. A 84, 063420 (2011).

[22] N. W. M. Ritchie, E. R. I. Abraham, Y. Y. Xiao, C. C. Bradley, R. G. Hulet, and P. S. Julienne, Phys. Rev. A 51, R890 (1995).

[23] The uv light source is a TA/DL-SHG 110 laser, manufactured by Toptica Photonics.

[24] D. A. Butts and D. S. Rokhsar, Phys. Rev. A 55, 4346 (1997).

[25] W. Ketterle and M. W. Zwierlein, Riv. Nuovo Cimento 31, 247 (2008)

[26] X. Du, Y. Zhang, and J. E. Thomas, Phys. Rev. Lett. 102, 250402 (2009).

[27] K. M. O'Hara, S. R. Granade, M. E. Gehm, and J. E. Thomas, Phys. Rev. A 63, 043403 (2001).

[28] T. Ido, Y. Isoya, and H. Katori, Phys. Rev. A 61, 061403 (2000).

[29] Ch. Grain, T. Nazarova, C. Degenhardt, F. Vogt, Ch. Lisdat, E. Tiemann, U. Sterr, and F. Riehle, Eur. Phys. J. D 42, 317 (2007).

[30] M. Safronova (private communication).

[31] T. A. Corcovilos, S. K. Baur, J. M. Hitchcock, E. J. Mueller, and R. G. Hulet, Phys. Rev. A 81, 013415 (2010). 